\title{
Investigating the pharmacological mechanisms of SheXiang XinTongNing against coronary heart disease based on network pharmacology
}

\section{Li-ying Jia}

China Pharmaceutical University

Jia Li

China Pharmaceutical University

\section{Gui-yun Cao}

Institute of Traditional Chinese Medicine, Shandong Hongjitang Pharmaceutical Group Co.

\section{Zhao-qing Meng}

Institute of Traditional Chinese Medicine, Shandong Hongjitang Pharmaceutical Group Co.

\section{Lu Gan}

China Pharmaceutical University

Jin-xin Li

Institute of Traditional Chinese Medicine, Shandong Hongjitang Pharmaceutical Group Co.

\section{Xin-yi Lan}

China Pharmaceutical University

\section{Chao-lin Yang}

Institute of Traditional Chinese Medicine, Shandong Hongjitang Pharmaceutical Group Co.

Chun-Feng Zhang ( $\square$ cpuzcfktz@163.com )

China Pharmaceutical University

\section{Research}

Keywords: Coronary heart disease, Network pharmacology, Pharmacological mechanisms

Posted Date: April 12th, 2020

DOI: https://doi.org/10.21203/rs.3.rs-21814/v1

License: (c) (i) This work is licensed under a Creative Commons Attribution 4.0 International License.

Read Full License 


\section{Abstract}

Background SheXiang XinTongNing, a commercially available Chinese patent medicine, has been widely used in the treatment of coronary heart disease. However, the mechanisms of SheXiang XinTongNing are still unclear. The aim of this study was to investigate the pharmacological mechanisms of SheXiang XinTongNing against coronary heart disease via network analysis.

Method The traditional Chinese medicine system pharmacology analysis platform was used to screen the potential active constituents of the six traditional Chinese medicines in SheXiang XinTongNing, and the potential targets were obtained from PharmMapper. The genome annotation database platform was used to screen the candidate targets related to coronary heart disease. Then the drug-components-targets network and protein interaction network were built by Cytoscape 3.6.0 software. Further, Go biofunctional enrichment analysis and KEGG pathway enrichment analysis were performed through annotation, visualization and integrated discovery database.

Results Results showed that the drugs-components-targets network contains 104 targets and 62 key components. The protein interaction network consisted of 107 nodes; key targets included Bcl2I1, IGF1, SRC, CASP3, et al. Functionally, the candidate targets were significantly associated with multiple pathways such as PI3K-Akt signaling pathway, MAPK signaling pathway, Ras signaling pathway, FoxO signaling pathway, Endocrine resistance. Given the above, the pharmacological activities of SheXiang XinTongNing may be predominantly related to several factors such as cell apoptosis, inflammation and angiogenesis.

Conclusion XTN can effectively attenuate the symptoms of coronary heart disease through diverse pathways. The research proves that network pharmacology can successfully reveal the mechanisms of traditional Chinese medicine in a holistic view. Our systematic analysis lays a foundation for further studying.

\section{Background}

Cardiovascular disease is a leading cause of death worldwide that caused 17.3 million deaths globally[1]. As one of the most important cardiovascular diseases, coronary heart disease(CHD) causes the most death and disability. It represents a substantial societal burden in developed countries and accounts for one-third of all deaths in the adult population[2,3]. The mortality of CHD is supposed to increase in developing countries from approximately 9 million in 1990 to about 19 million by 2020[4]. Notably, a new study suggested that patients with cardiovascular disease are more likely to be infected with COVID-19[5]. Data from the National Health Commission showed that 17 percent of COVID-19 patients had the comorbidity of $\mathrm{CHD}[6]$. High blood pressure, cholesterol levels, diabetes, obesity have been considered as major determining factors of $\mathrm{CHD}$ [7]. Women with onset of type 1 diabetes before age 10 have a 60 -times increased risk of $\mathrm{CHD}[8]$. In addition, life behaviors, such as alcohol intake and cigarette consumption[9, 10], environmental toxic metal contaminants[11] and the degree of ApoB are also related to the augment 
of CHD data[12]. Apart from those, it is reported that HIV infection caused a substantially increased risk of $\mathrm{CHD}$ [13]. Currently, modification of lifestyles, percutaneous coronary intervention (PCl), coronary artery bypass grafting (CABG) and medication therapy such as nitrates, beta receptor-blockers, stains, antiplatelet drugs are the mainstream therapeutic methods for CHD. Nevertheless, there are many limitations in the treatment of $\mathrm{CHD}$, such as side effects of drugs especially Western drugs, lack of comprehensive management, patients' resistance to changing lifestyles[14].

Traditional Chinese medicine (TCM), originated from China, which is characterized by its minor side effects and synergistic therapeutic efficacies, has been long used in clinical practice and plays indispensable roles in various diseases. In particular, it has broadened the ideas of therapeutic approaches and achieved crucial effects in $\operatorname{CHD}[15,16]$. According to the TCM theory, the pathogenesis of coronary heart disease may be phlegm turbidity, cold coagulation, qi stagnation and blood stasis[17]. At present, there are many TCM preparations and Chinese patent drugs for relieving chest congestion, activating Qi and promoting blood circulation for removing blood stasis, which show remarkable effects in CHD. Particularly, SheXiang XinTongNing(XTN), a commercially available Chinese patent medicine which has been widely used in clinical treatment of CHD. XTN is composed of 6 herbs, including Moschus (Shexiang, SX), Ligusticum chuanxiong (Chuanxiong, CX), Rhizoma Corydalis(Yanhusuo, YHS), Panax ginseng(Renshen, RS), Borneolum Syntheticum(Bingpian, BP) and Styrax(Suhexiang, SHX). Among them, $\mathrm{SX}$ is the principal drug and plays a role in resuscitation; $\mathrm{CX}$ mainly promotes blood circulation and activates qi; YHS principally regulates qi-flowing for relieving pain. The combination of $\mathrm{CX}$ and YHS are the assistant drugs, playing a prominent role in improving the two essentials (qi and blood) of human bodies to alleviate the diseases; BP is the adjuvant drug which exerts functions in activating brain and clearing away for resuscitation. In addition, it enhances the effects of principal and assistant drugs. The formulae conforms to the theory of TCM and the six kinds of herbs have a synergistic effect, so as to achieve a prominent efficacy in relieving CHD. However, it is precisely because of the multiple components and multiple targets of TCM that the mechanism of TCM is difficult to be clarified. Although XTN has been applied in clinic for a long time, the underlying mechanisms of XTN are still vague.

Network Pharmacology is a systematic method to study the mechanisms of drugs and diseases based on the network construction of drugs, constituents, genes, protein targets, and diseases[18]. It coincides well with the holistic ideas of 'network target, multicomponent therapeutics' of TCM and contributes greatly to the prediction of potential drugs and the screening of the components, targets as well as pathways of drugs[19]. In previous study, the network pharmacology has been used in elucidating the key components and mechanisms of TCM involved in their therapeutic effects, such as the mechanisms of Erxian Decoction against TNF-a induced osteoblast apoptosis[20], the mechanisms of Huayu-QiangshenTongbi formula on rheumatoid arthritis[21], the anticancer mechanisms of Compound Kushen Injection against hepatocellular carcinoma[22].

In this study, a network pharmacology approach was adopted to illuminate the underlying mechanisms of XTN against CHD through following steps: (1) Screening the chemical components of the six herbs 
contained in XTN; (2) Predicting the candidate targets of XTN related to CHD; (3) Illustrating a drugcomponents-targets network and a protein interaction(PPI) network; (4) Functional analysis of XTN for investigating the mechanisms of XTN acting on CHD.

As a consequence, our study could provide novel insights into the mechanisms of XTN treating CHD. More generally, offers support for further study. The flowchart of our study was shown in Fig. 1.

\section{Methods}

\section{Active components screening}

TCMSP(traditional Chinese medicine system pharmacology analysis platform) is a specialized computational platform for systematic pharmacology-based analysis, which contains the information of herbs. The identities of the chemical components in YHS, SX, CX, RS, BP, SHX were retrieved from TCMSP database (http://tcmspw.com/)[23]. The oral availability (OB) and drug-likeness (DL) are considered as two key indicators in drug screen. The $\mathrm{OB}$ index represents the percentage of drugs reaching the circulation after oral administration, and the $\mathrm{DL}$ index is a qualitative parameter that estimate the similarity between a substance and an existing drug[24]. The OB threshold was set at $40 \%(\mathrm{OB}>40 \%)$ and the threshold of $D L$ was set at $0.18(D L \geq 0.18)$, by which the active ingredients were selected. Since SX has not been detected in the database, and there are some compounds with high content or strong activity but low $\mathrm{OB}$ or $\mathrm{DL}$ in the above five herbs reported in the literature, thus, tetrahydropalmatine, tetrandrine, tetramethylolactone, ligustilide, ligustrazine, ginsenosides (Rb1, Rg1, Rg3, Rd, Re), muscone, cinnamaldehyde and Bienyl benzoate in XTN were also incorporate in the study. The 3D structures of each active component were retrieved from Pubchem (https://pubchem.ncbi.nlm.nih.gov/) [25]and saved in .sdf format.

\section{Putative and Candidate targets of drugs}

In addition, the putative targets hitting every components of XTN were obtained through PharmMapper (http://www.lilab-ecust.cn/pharmmapper/) database[26]. PharmMapper is one of the reverse molecular docking methods developed by reverse pharmacophore mapping based on ligand features, which uses active small molecules as probes to search for potential drug targets and thus predict the biological activity of small molecules. The program can quickly obtain drug target information by retrieving four databases, Target Bank, Drug Bank, Binding DB and PDTD[27]. Due to its advantages of fast operation speed and comprehensive target information, it has been extensively used in the research of TCM targets. Firstly, the .sdf format file of the active components of XTN was uploaded into PharmMapper, then we used the reverse pharmacophore matching method to get the virtual screening results. The active small molecules were used as probes to search for potential drug targets. When the docking score between molecules and targets, that is, the molecular-target matching degree (Fit Score) is greater than 4.5 , it is considered that the target interacts with the chemical components in XTN, thus the putative target is screened out. 
The PDB ID of targets was converted into the Gene name with UniProt database(http://www.uniprot.org/) [28]. Subsequently, the target genes related to coronary heart disease were collected with "coronary heart disease" as key words in genome annotation database platform(Genecards) (https://www.genecards.org/) [29]database, and the common targets were screened as the candidate targets of XTN.

\section{Network construction}

To investigate the relationship of XTN and CHD, the drug-components-targets network and protein interaction(PPI) network were constructed with Cytoscape 3.6.0 software(https://cytoscape.org/)[30]. In the drugs-components-targets graphical network, the drug, the active drug molecules and the candidate targets refer to the nodes of network; the edges, which connected the nodes refer to the interactions, and the number of edges in the network refers to the node values.

Further, in order to clarify the interaction between the potential target proteins of XTN, the PPI network model of the screened target proteins was constructed on STRING platform(http://string-db.org/)[31]. The protein type was set to "Homo sapiens" (human) for operation, the lowest interaction threshold was set to medium "medium confidence", and other parameters were kept at the default setting. Screen the top 30 core targets, then the PPI network model was imported into Cytoscape 3.6. 0 software, the Network Analysis function was used for analysis, and the size of the node was adjusted in accordance with the connection degrees ( the greater the connection degree is, the closer the node is related to other nodes, and the more important it is in the network), and the PPI network diagram was established.

\section{Pathway enrichment analysis and biological functional analysis}

The annotation, visualization and integrated discovery database (DAVID) database(https://david.ncifcrf.gov/)[32] is a biological information database that integrates biological data and analytical tools together, which can be used for pathway analysis and biological function analysis. The KEGG signal pathway enrichment analysis and GO biological process enrichment of the candidate targets of XTN were carried out by DAVID database. The biological processes and pathways with $P<0.01$ were selected and sorted in line with the number of enriched genes from large to small, then the top 20 biological processes and pathways were chosen for visualization analysis.

\section{Results}

\section{Active compounds in XTN}

Retrieved from TCMSP database, the components of the 6 herbs in XTN were collected. On the basis of threshold value of $\mathrm{OB}>40 \%$ and $\mathrm{DL} \geq 0.18,62$ active compounds were selected as shown in Table 1 . 
Table 1

Active compounds of 6 herbs and their $\mathrm{OB}$ and $\mathrm{DL}$

\begin{tabular}{|c|c|c|c|c|}
\hline Compound & herb & Chemical & OB & DL \\
\hline C01 & YHS1 & Cryptopin & 78.74 & 0.72 \\
\hline C02 & YHS2 & Dihydrosanguinarine & 59.31 & 0.86 \\
\hline $\mathrm{CO3}$ & YHS3 & (R)-Canadine & 55.37 & 0.77 \\
\hline $\mathrm{CO4}$ & YHS4 & Hyndarin & 73.94 & 0.64 \\
\hline C05 & YHS5 & Capaurine & 62.91 & 0.69 \\
\hline $\mathrm{C06}$ & YHS6 & Clarkeanidine & 86.65 & 0.54 \\
\hline $\mathrm{C} 07$ & YHS7 & Corydaline & 65.84 & 0.68 \\
\hline $\mathrm{C08}$ & YHS8 & Corydalmine & 52.5 & 0.59 \\
\hline C09 & YHS9 & Corynoline & 46.06 & 0.85 \\
\hline C10 & YHS10 & $\begin{array}{l}\text { methyl-2-(3,4,6,7-tetramethoxyphenanthren-1- } \\
\text { yl)ethanamine }\end{array}$ & 61.15 & 0.44 \\
\hline C11 & YHS11 & Dehydrocorybulbine & 46.97 & 0.63 \\
\hline C12 & YHS12 & dehydrocorydaline & 41.98 & 0.68 \\
\hline C13 & YHS13 & Dehydrocorydalmine & 43.9 & 0.59 \\
\hline C14 & YHS14 & Fumaricine & 43.95 & 0.72 \\
\hline C15 & YHS15 & isocorybulbine & 40.18 & 0.66 \\
\hline C16 & YHS16 & leonticine & 45.79 & 0.26 \\
\hline C17 & YHS17 & 13-methylpalmatrubine & 40.97 & 0.63 \\
\hline C18 & YHS18 & $\mathrm{N}$-methyllaurotetanine & 41.62 & 0.56 \\
\hline C19 & YHS19 & Pseudoprotopine & 53.75 & 0.83 \\
\hline C20 & YHS20 & saulatine & 42.74 & 0.79 \\
\hline $\mathrm{C} 21$ & YHS21 & stylopine & 48.25 & 0.85 \\
\hline $\mathrm{C} 22$ & YHS22 & tetrahydroprotopapaverine & 57.28 & 0.33 \\
\hline $\mathrm{C} 23$ & YHS23 & $\begin{array}{l}\text { 2,3,9,10-tetramethoxy-13-methyl-5,6- } \\
\text { dihydroisoquinolino[2,1-b]isoquinolin-8-one }\end{array}$ & 76.77 & 0.73 \\
\hline $\mathrm{C} 24$ & YHS24 & Stigmasterol & 43.83 & 0.76 \\
\hline $\mathrm{C} 25$ & YHS25 & palmatine & 64.6 & 0.65 \\
\hline
\end{tabular}




\begin{tabular}{|c|c|c|c|c|}
\hline Compound & herb & Chemical & OB & $\mathrm{DL}$ \\
\hline C26 & YHS26 & Fumarine & 59.26 & 0.83 \\
\hline $\mathrm{C} 27$ & YHS27 & bicuculline & 69.67 & 0.88 \\
\hline $\mathrm{C} 28$ & YHS28 & Bulbocapnine & 47.54 & 0.69 \\
\hline C29 & YHS29 & quercetin & 46.43 & 0.28 \\
\hline C30 & YHS30 & Isocorypalmine & 35.77 & 0.59 \\
\hline C31 & YHS31 & columbamine & 26.94 & 0.59 \\
\hline C32 & CX1 & Mandenol & 42 & 0.19 \\
\hline C33 & $\mathrm{cX} 2$ & Myricanone & 40.6 & 0.51 \\
\hline C34 & $\mathrm{CX} 3$ & Perlolyrine & 65.95 & 0.27 \\
\hline C35 & CX4 & senkyunone & 47.66 & 0.24 \\
\hline C36 & CX5 & wallichilide & 42.31 & 0.71 \\
\hline C37 & CX6 & FA & 68.96 & 0.71 \\
\hline C38 & $\mathrm{CX7}$ & Senkyunolide A & 26.56 & 0.07 \\
\hline C39 & CX8 & (Z)-Ligustilide & 53.72 & 0.07 \\
\hline $\mathrm{C} 40$ & CX9 & tetramethylpyrazine & 20.01 & 0.03 \\
\hline C41 & RS1 & Diop & 43.59 & 0.39 \\
\hline C42 & RS2 & Inermin & 65.83 & 0.54 \\
\hline $\mathrm{C} 43$ & RS3 & kaempferol & 41.88 & 0.24 \\
\hline C44 & RS4 & Aposiopolamine & 66.65 & 0.22 \\
\hline C45 & RS5 & Celabenzine & 101.88 & 0.49 \\
\hline C46 & RS6 & Dianthramine & 40.45 & 0.2 \\
\hline C47 & RS7 & arachidonate & 45.57 & 0.2 \\
\hline C48 & RS8 & Frutinone A & 65.9 & 0.34 \\
\hline C49 & RS9 & Girinimbin & 61.22 & 0.31 \\
\hline C50 & RS10 & malkangunin & 57.71 & 0.63 \\
\hline C51 & RS11 & suchilactone & 57.52 & 0.56 \\
\hline C52 & RS12 & ginsenoside Rb1 & -- & -- \\
\hline C53 & RS13 & ginsenoside Rg1 & -- & -- \\
\hline
\end{tabular}




\begin{tabular}{|lllll|}
\hline Compound & herb & Chemical & OB & DL \\
\hline C54 & RS14 & ginsenoside Rg3 & -- & -- \\
\hline C55 & RS15 & ginsenoside Rd & -- & -- \\
\hline C56 & RS16 & ginsenoside Re & -- & -- \\
\hline C57 & SX1 & Muscone & -- & -- \\
\hline C58 & BP1 & asiatic acid & -- & -- \\
\hline C59 & BP2 & bronyl acetate & 41.38 & 0.71 \\
\hline C60 & BP3 & dipterocarpol & 59.3 & 0.51 \\
\hline C61 & SHX1 & cinnamaldehyde & 41.71 & 0.76 \\
\hline C62 & SHX2 & BENZYL BENZOATE & 31.99 & 0.02 \\
\hline
\end{tabular}

\section{Candidate targets screening}

As results, a total of 831 putative targets were screened from PharmMapper. Among them, there were 341 putative targets for YHS, 212 for CX, 182 for RS, 11 for SX, 71 for BP and 12 for SHX. Further, we obtained 104 candidate targets connected with CHD from Genecards platform, among which the top ten in terms of degree included TTR, PIPN1, AKR1C3, PDE4D, HSP90AA1, FKBP1A, MME, MAPK14, VDR and CA2.

\section{Construction of drug-components-targets network}

In addition, we generated drug-components-targets network on the basis of Cytoscape 3.6.0 software. As shown in Fig. 2, the network comprises of 171 nodes, the nodes in yellow are the six herbs in XTN, the nodes in blue are the chemical components of drugs and the nodes in red are the candidate targets.

\section{Construction of PPI network}

The STRING database was used to construct PPI network model. Then the model was introduced into Cytoscape 3.6. 0 software to produce PPI network diagram(Fig. 3). As shown in Fig. 4, the top 30 core proteins include ALB, IGF1, SRC, CASP3, EGFR, ESR1,HRAS, HSP90AA1, MAPK14, STAT1, Bcl2I1, NOS3, AR, IL2, NR3C1, PPARG, KDR, MAP2K1, ACE, PGR, ABL1, F2, CCNA2, GSK3B, CDK2, LCK, PTPN1, REN, CDKN1B and KNT. .

\section{Functional analysis of candidate targets}

As exhibited in Fig. 5, the candidate targets of XTN were involved in numerous biological functions, including steroid hormone receptor activity, nuclear receptor activity, transcription factor activity, director ligand regulated sequence-specific binding, steroid binding, monocarboxylic acid binding, nuclear receptor coactivator activity, protein tyrosine kinase activity, carboxylic acid binding, transmembrane receptor protein kinase activity, organic acid binding, fatty acid binding, transcription coactivator activity, 
transmembrane receptor protein tyrosine kinase activity, hormone binding, endopeptidase activity, phosphatase binding, protein phosphatase binding, DNA-binding transcription activator activity, RNA polymerase $₫$-specific, $\mathrm{SH} 2$ domain binding and nuclear hormone receptor binding.

In addition, the KEGG pathway enrichment shed light on the pathways that the candidate targets involved in. As shown in Fig. 6, the treatment of XTN against CHD was closely associated with PI3K-AKt signaling pathway, MAPK signaling pathway, Ras signaling pathway, Rap1 signaling pathway, EGFR tyrosine kinase inhibitor resistance, FoxO signaling pathway, Th17 cell differentiation, Endocrine resistance, etc.

\section{Discussion}

TCM refers to a natural healthcare, holistic system based on over 2000 years of principles and practice which takes all aspects of patients' life into account rather than just apparent symptoms[33,34]. As the saying goes, "treatment aiming at its pathogenesis" and "treating both manifestation and root cause of disease". TCM prescriptions are usually made up of several herbs to play a synergistic role. At the same time, this also makes it difficult to interpret the mechanism of TCM, hindering its widespread clinic application in the world[35]. Network pharmacology connects the main, supplementary and auxiliary therapeutic components of traditional Chinese medicine prescriptions with the main, supplementary and auxiliary targets in the disease network[36]. In the present study, the network pharmacology approach offers an effective channel for uncovering the mechanisms of XTN including its active components and CHD-related targets. Firstly, we focused on the 62 candidate targets. Secondly, drug-components-targets network were constructed. Thirdly, the PPI network was constructed.

Subsequently, we analyzed the mechanism of XTN by mapping the candidate targets onto the pathway enrichment.

XTN is a classic TCM formula composing of 6 herbs with considerable effects on CHD. After OB and DL filtering, 62 bioactive compounds were obtained from TCMSP databases that is commonly adopted[37]. The pharmacological activities of these components in CHD have been reported previously. For example, Dehydrocorydalin, an active component of YHS, is known for its anti-platelet, anti-inflammatory and antimyocardial hypoxia activities[38]; Hyndarin is a natural alkaloid isolated from YHS, which has been proved to exert various cardiovascular effects, namely anti-apoptosis, anti-oxidant, cardioprotection and improving cerebral ischemia-reperfusion injuries[39-41]. In addition, Muscone is the active compound of SX, several studies have shown that it has anti-cerebral ischemia and anti-myocardial ischemia activities. Accordingly, this indicates that multiple components of XTN function through multiple targets.

There are 104 overlapping targets genes between XTN and CHD. Besides, we obtained the top 10 genes from the drug-components-targets network according to the degree value, which may be the key genes in the treatment of CHD. Moreover, we get the core proteins of interests, Casp3 and Bcl2l1 from PPI network. Casp3 is closely related to apoptosis and plays critical role in apoptotic pathway[42]. One of the earliest changes of apoptosis is characterized by the emergence of a series of proteases - Caspases. Activated caspases cleaves many intracellular enzymes and causes morphological changes of apoptotic cells; the 
core of this process is exactly the activation of Casp-3[43]. Bcl2/1 is an anti-apoptotic protein, which is a downstream protein in the PI3K-Akt pathway. Based on the results of biological functional analysis, there are a variety of pathways like PI3K-Akt signaling pathway, MAPK signaling pathway, FoxO signaling pathway[44], Ras signaling pathway[45] and Endocrine resistance[46], are robustly correlated with the pathogenesis of CHD. Interestingly, the PI3K-AKT signaling pathway has been reported to participate in the signal transduction that related to various cell activities such as proliferation, differentiation and apoptosis[47]. It balances the pro-apoptotic (Bax, Bad, Bcl-xs) and anti-apoptotic (Bcl-2, Bc1-xl) in Bcl-2 family via activation of caspases and the Bcl-2 family, thereby tightly regulates cell apoptosis as shown in Fig. 7[48]. Meanwhile, as the members of Bcl-2 family, Bcl-2 and Bcl-xl are also significant anti-oxidant proteins that play a vital role in scavenging free radicals and reducing the production of superoxides[49]. Zhu et al. demonstrated that the up-regulation of Bcl2 expression in PI3K-Akt pathway can effectively protect the injury caused by ischemia and hypoxia[50]. Apart from this, the MAPK pathway, which is relevant with the occurrence of various pathological processes, mediates a variety of cell functions, such as proliferation, differentiation, transformation and apoptosis through phosphorylation of cytoskeletal proteins and nuclear transcription factor [51]. A study has shown that the activation of MAPK pathway may stimulate the release of some inflammatory mediators, specifically MMP9 and IL-6, which leads to the process of atherosclerosis[52]. Additionally, Yuan et al. [53]believed that the up-regulation of MAPK pathways could promote angiogenesis. In general, the effect of XTN against CHD may achieve by regulating the expression of apoptosis factors, inflammatory mediators and via multiple pathways such as PI3K-Akt pathway and MAPK pathway.

It seems that the network pharmacology approach is suitable for deciphering the mechanisms of CHD. The utilization of systematic pharmacology may broaden our ideas in CHD medication therapy. However, there are still some limitations in our study. On the one hand, our research is on the basis of virtual screen and prediction that is lack of experimental support. On the other hand, the process of OB and DL screening is more likely to cause data missing of some constituents with lower OB or DL value but stronger activities. In response to these problems, In future research, further experiment validation will be conducted to verify the pathways and mechanism of XTN by in vitro and in vivo experiments.

\section{Conclusion}

In summary, the mechanism of XTN treating for CHD was analyzed systematically based on network pharmacology in this study. It offers new insights into exploring of the mechanisms underlying effects of XTN on CHD. We collected the active compounds and candidate targets of XTN, established a drugcomponent-targets network and a PPI network and obtained the pathways relating to $\mathrm{CHD}$. The active components and targets of XTN are distributed in different pathways which can play a synergistic role in the prevention and treatment of CHD. Target proteins combining with pathway enrichment analyses indicated that XTN exerted its anti-CHD effect by regulating multiple pathways, including FoxO signaling pathway, Ras signaling pathway, Endocrine resistance, PI3K-Akt mediated cell apoptosis pathway, MAPK mediated anti-inflammatory and angiogenesis pathways and so forth. This study presents a fast, 
economical and comprehensive method for the studies of XTN from a holistic view, which lays a foundation for further study and clinic utility.

\section{Abbreviations}

XTN: SheXiang XinTongNing; CHD: coronary heart disease; PCl: percutaneous coronary intervention; CABG :coronary artery bypass grafting; TCM: Traditional Chinese medicine; SX :Moschus (Shexiang); CX :Ligusticum chuanxiong (Chuanxiong); YHS: Rhizoma Corydalis(Yanhusuo); RS :Panax ginseng(Renshen); BP :Borneolum Syntheticum(Bingpian); SHX: Styrax(Suhexiang);TCMSP: tradiational Chinese medicine system pharmacology analysis platform; $\mathrm{OB}$ :oral availability; DL:drug-likeness ; DAVID: annotation, visualization and integrated discovery database

\section{Declarations}

\section{Acknowledgements}

Not applicable.

\section{Authors' contributions}

Z-QM and JL conceived and designed the study. GL and JL collected the data. J-XL and X-YL performed the data analysis, L-YJ and G-YC wrote the manuscript. All authors are responsible for reviewing data. All authors read and approved the fnal manuscript.

\section{Funding}

This work was financially supported by National Key R\&D Program of China (2018YFC1707105), and The Funding of Double First-rate Discipline Innovation Team (CPU2018GF05).

\section{Availability of data and materials}

The datasets used and analyzed during the current study are available from the corresponding author on reasonable request.

The datasets generated and/or analysed during the current study are available in the TCMSP, http://tcmspw.com/

Pubchem https://pubchem.ncbi.nlm.nih.gov/

PharmMapper http://www.lilab-ecust.cn/pharmmapper/

UniProt http://www.uniprot.org/

Genecards https://www.genecards.org/ 
Cytoscape 3.6.0 https://cytoscape.org/

STRING http://string-db.org/

DAVID https://david.ncifcrf.gov/

Ethics approval and consent to participate

Not applicable.

\section{Consent for publication}

The manuscript is approved by all authors for publication.

\section{Competing interests}

The authors declare that they have no competing interests.

\section{Authors details}

${ }^{1}$ School of Traditional Chinese Pharmacy, China Pharmaceutical University, Nanjing, 210009, China. ${ }^{2}$ Institute of Traditional Chinese Medicine, Shandong Hongjitang Pharmaceutical Group Co., Ltd. Jinan, 250103, China

\section{References}

1. Townsend N, Wilson L, Bhatnagar P, Wickramasinghe K, Rayner M. Nichols M.Cardiovascular disease in europe: epidemiological update 2016. Eur Heart J. 2016;37(42):3232-45.

2. Roger VL. Epidemiology of myocardial infarction. Med Clin North Am. 2007;91(4):537-52.

3. Letnes JM, Dalen H, Vesterbekkmo EK, Wisløff U, Nes BM. Peak oxygen uptake and incident coronary heart disease in a healthy population: the hunt fitness study. Eur Heart J. 2019;40(20):1633-9.

4. Sanchis-Gomar F, Perez-Quilis C, Leischik R, Lucia A. Epidemiology of coronary heart disease and acute coronary syndrome. Annals of translational medicine. 2016;4(13):256.

5. Kevin J, Clerkin JM, Griffin D, Burkhoff, et al. Coronavirus Disease 2019 (COVID-19) and Cardiovascular Disease.Circulation. 2020.

6. Zheng YY, Ma YT, Zhang JY, Xie X. COVID-19 and the cardiovascular system. Nat Rev Cardiol. 2020.

7. Timmis A, Townsend N, Gale C, Grobbee R, Maniadakis N, Flather M, et al. European society of cardiology: cardiovascular disease statistics 2017. Eur Heart J. 2018;39(7):508-79.

8. Rawshani A, Sattar N, Franzén S, Rawshani A, Hattersley AT, Svensson A, Eliasson B, Gudbjörnsdottir $\mathrm{S}$. Excess mortality and cardiovascular disease in young adults with type 1 diabetes in relation to age at onset: a nationwide, register-based cohort study. The Lancet. 2018;392(10146):477-86. 
9. Hackshaw A, Morris JK, Boniface S, Tang JL, Milenkovic D. Low cigarette consumption and risk of coronary heart disease and stroke: meta-analysis of 141 cohort studies in 55 study reports. BMJ. 2018;360:j5855.

10. Ricci C, Wood A, Muller D, Gunter MJ, Agudo A, Boeing H, et al. Alcohol intake in relation to non-fatal and fatal coronary heart disease and stroke: epic-cvd case-cohort study. BMJ. 2018;361:k934.

11. Chowdhury R, Ramond A, O Keeffe LM, Shahzad S, Kunutsor SK, Muka T, Gregson J, Willeit P, Warnakula S, Khan $\mathrm{H}$,et al. Environmental toxic metal contaminants and risk of cardiovascular disease: systematic review and meta-analysis. BMJ. 2018:k3310.

12. Navar AM. The evolving story of triglycerides and coronary heart disease risk. JAMA. 2019;321(4):347-9.

13. Hsue PY, Waters DD. Hiv infection and coronary heart disease: mechanisms and management. Nat Rev Cardiol. 2019;16(12):745-59.

14. Mao X, Xu H, Li S, Su J, Li W, Guo Q, Wang P, Guo R, Xiao X, Zhang Y, Yang H. Exploring pharmacological mechanisms of xueshuan-xinmai-ning tablets acting on coronary heart disease based on drug target-disease gene interaction network. Phytomedicine. 2019;54:159-68.

15. Shujing Sheng JWHL. Network pharmacology analyses of the antithrombotic pharmacological mechanism of fufang xueshuantong capsule with experimental support using disseminated intravascular coagulation rats. J Ethnopharmacol. 2014;154(3):735-44.

16. Tomioka $\mathrm{H}$. Usefulness of chinese herbal medicines as host-directed therapeutics against mycobacterial infections: a review. The American Journal of Chinese Medicine. 2017;45(08):1597611.

17. Yang Y, Huang C, Su X, Zhu J, Chen X, Fu Y, Wang Z, Zhou J, Xiao W, Zheng C, Wang Y. Deciphering the multicomponent synergy mechanism from a systems pharmacology perspective: application to gualou xiebai decoction for coronary heart disease. Journal of Functional Foods. 2018;47:143-55.

18. Chen L, Cao Y, Zhang H, Lv D, Zhao Y, Liu Y, Ye G, Chai Y. Network pharmacology-based strategy for predicting active ingredients and potential targets of yangxinshi tablet for treating heart failure. $J$ Ethnopharmacol. 2018;219:359-68.

19. Yu G, Luo Z, Zhou Y, Zhang L, Wu Y, Ding L, Shi Y. Uncovering the pharmacological mechanism of carthamus tinctorius I. On cardiovascular disease by a systems pharmacology approach. Biomed Pharmacother. 2019;117:109094.

20. Wang N, Xin H, Xu P, Yu Z, Shou D. Erxian decoction attenuates tnf-a induced osteoblast apoptosis by modulating the akt/nrf2/ho-1 signaling pathway. Front Pharmacol. 2019;10:988.

21. Wang Z, Linghu KG, Hu Y, Zuo H, Yi H, Xiong SH, Lu J, Chan G, Yu H, Huang RY. Deciphering the pharmacological mechanisms of the huayu-qiangshen-tongbi formula through integrating network pharmacology and in vitro pharmacological investigation. Front Pharmacol. 2019;10:1065.

22. Gao L, Wang KX, Zhou YZ, Fang JS, Qin XM, Du GH. Uncovering the anticancer mechanism of compound kushen injection against hcc by integrating quantitative analysis, network analysis and experimental validation. Sci Rep. 2018;8(1):624. 
23. Sun JH, Sun F, Yan B, Li JY, Xin DL. Data mining and systematic pharmacology to reveal the mechanisms of traditional chinese medicine in mycoplasma pneumoniae pneumonia treatment. Biomed Pharmacother. 2020;125:109900.

24. Huang J, Guo W, Cheung F, Tan HY, Wang N, Feng Y. Integrating Network Pharmacology and Experimental Models to Investigate the Efficacy of Coptidis and Scutellaria Containing Huanglian Jiedu Decoction on Hepatocellular Carcinoma. The American Journal of Chinese Medicine. 2020;48(1):1-22.

25. Zhang R, Zhu X, Bai H, Ning K. Network pharmacology databases for traditional chinese medicine: review and assessment. Front Pharmacol. 2019;10:123.

26. Zhang J, Liang R, Wang L, Yang B. Effects and mechanisms of danshen-shanzha herb-pair for atherosclerosis treatment using network pharmacology and experimental pharmacology. $J$ Ethnopharmacol. 2019;229:104-14.

27. Liu X, Ouyang S, Yu B, Liu Y, Huang K, Gong J, Zheng S, Li Z, Li H, Jiang H. Pharmmapper s erver: a web server for potential drug target identification using pharmacophore mapping approach. Nucleic Acids Res. 2010;38(suppl_2):W609-14.

28. Wu R, Jiang B, Li H, Dang W, Bao W, Li H, Ye G, Shen X. A network pharmacology approach to discover action mechanisms of yangxinshi tablet for improving energy metabolism in chronic ischemic heart failure. J Ethnopharmacol. 2020;246:112227.

29. Zeng L, Yang K, Ge J. Uncovering the pharmacological mechanism of astragalus salvia compound on pregnancy-induced hypertension syndrome by a network pharmacology approach. Sci Rep. 2017;7(1):16849.

30. Chen W, Da W, Li C, Fan H, Liang R, Yuan J, Huang X, Yang R, Zhang J, Zhu J. Network pharmacology-based identification of the protective mechanisms of taraxasterol in experimental colitis. Int Immunopharmacol. 2019;71:259-66.

31. Zhao S, Liu Z, Wang M, He D, Liu L, Shu Y, Song Z, Li H, Liu Y, Lu A. Anti-inflammatory effects of zhishi and zhiqiao revealed by network pharmacology integrated with molecular mechanism and metabolomics studies. Phytomedicine. 2018;50:61-72.

32. Huang DW, Sherman BT, Lempicki RA. Bioinformatics enrichment tools: paths toward the comprehensive functional analysis of large gene lists. Nucleic Acids Res. 2009;37:1-13.

33. Zhang S, Su Z, Liu R, Diao Y, Li S, Ya-Ping-Hua, Li H, Zhang W. Exploring the pathways and t argets of shexiang baoxin pill for coronary heart disease through a network pharmacology approach. World Journal of Traditional Chinese Medicine. 2018;4(4):137-46.

34. Xu Q, Bauer R, Hendry BM, Fan T, Zhao Z, Duez P, Simmonds MSJ, Witt CM, Lu A, Robinson N, Guo D, Hylands PJ. The quest for modernisation of traditional chinese medicine. BMC Complement Altern Med. 2013;13:132.

35. Liu L, Du B, Zhang H, Guo X, Zhou Z, Xiu A, Liu C, Su S, Ai H. A network pharmacology approach to explore the mechanisms of erxian decoction in polycystic ovary syndrome. Chin Med. 2018;13:46. 
36. Yang M, Chen JL, Xu LW, Ji G. Navigating traditional chinese medicine network pharmacology and computational tools. Evid Based Complement Alternat Med. 2013;2013:731969.

37. Sundarrajan S, Arumugam M. A systems pharmacology perspective to decipher the mechanism of action of parangichakkai chooranam, a siddha formulation for the treatment of psoriasis. Biomed Pharmacother. 2017;88:74-86.

38. Tan CN, Zhang Q, Li CH, Fan JJ, Yang FQ, et al. Potential target-related proteins in rabbit platelets. Phytomedicine. 2018;7113(18):30485-9.

39. Han Y, Zhang W, Tang Y, Zhou SM, et al. L-tetrahydropalmatine, an active component of corydalisyanhusuo w.t. Wang, protects against myocardial ischaemia-reperfusion injury in rats. PLOS ONE. 2012;7(6):38627.

40. Wu L, Ling H, Li L, Jiang L, He M. Beneficial effects of the extract from corydalis yanhusuo in rats with heart failure following myocardial infarction. J Pharm Pharmacol. 2007;59(5):695-701.

41. Mao XW, Pan CS, Huang P, Liu YY, Wang CS, Yan L, Hu BH, Chang X, He K, Mu HN, Li Q, Sun K, Fan JY, Han JY. Levo-tetrahydropalmatine attenuates mouse blood-brain barrier injury induced by focal cerebral ischemia and reperfusion: involvement of src kinase. Sci Rep. 2015;5:11155.

42. Elham Ahmadiana, Solmaz Maleki Dizaja Elaheh. Effect of silver nanoparticles in the induction of apoptosis on human hepatocellular carcinoma (hepg2) cell line. Materials Science \& Engineering C. 2018;93(2018):465-471.

43. Reiner $\mathrm{U}$, Ja Nicke MLSM. Caspase-3 is required for DNA fragmentation and morphological changes associated with apoptosis. J Biol Chem. 1998;273:9357-60.

44. Ferdous A, Battiprolu PK, Ni YG, Rothermel BA, Hill JA. FoxO, autophagy, and cardiac remodeling. J Cardiovasc Transl Res. 2010;3(4):355-64.

45. Maria A, Manush Saydmohammed1, Daniel A, et al. Dusp6 attenuates Ras/MAPK signaling to limit zebrafish heart regeneration. Development. 2018;145(5).

46. Pino AD, DeFronzo RA. Insulin resistance and atherosclerosis: Implications for insulin-sensitizing agents. Endocr Rev. 2019;40(6):1447-67.

47. Deng S, Dai G, Chen S, Nie Z, Zhou J, Fang H, Peng H. Dexamethasone induces osteoblast apoptosis through ros-pi3k/akt/gsk3 $\beta$ signaling pathway. Biomedicine \& Pharmacotherapy. 2019;110(2019):602-608.

48. Zhou BH, Tan PP, Jia LS, Zhao WP, et al. PI3K-Akt signal pathway involment in fluoride-induced apoptosis in C2C12 cells. Chemosphere. 2018;199:297-302.

49. Niture SK, Jaiswal AK. Nrf2 protein up-regulates anti-apoptotic protein $\mathrm{Bcl}-2$ and prevents cellularapoptosis. J Biol Chem. 2012;287(13):9873-86.

50. Zhu HL, Zhang YS, Shi ZS, Lu D, Li TT, et al. The neuroprotection of Liraglutide against ischaemiainduced apoptosis through the activation of the PI3K/Akt and MAPK Pathways. Sci Rep. 2016;6:26859. 
51. Berra E, Milanini J, Richard DE, Le Gall M, Vinal F, et al. Signaling angiogenesis via p42/p44 MAP kinase and hypoxia. Biochem Pharmacol. 2000;60(8):1171-8.

52. Li Z, Yang L, Yu CQ, et al. Anti-inflammatory and antioxidative effects of Dan-Lou tablets in the treatment of coronary heart disease revealed by metabolomics integrated with molecular mechanism studies. Journal of Ethnopharmacology. 2019;240(2019):111911.

53. Yuan X, Han L, et al. Cinnamaldehyde accelerates wound healing by promoting angiogenesis via upregulation of PI3K and MAPK signaling pathways. Lab Invest. 2018;98(6):783-98.

\section{Figures}

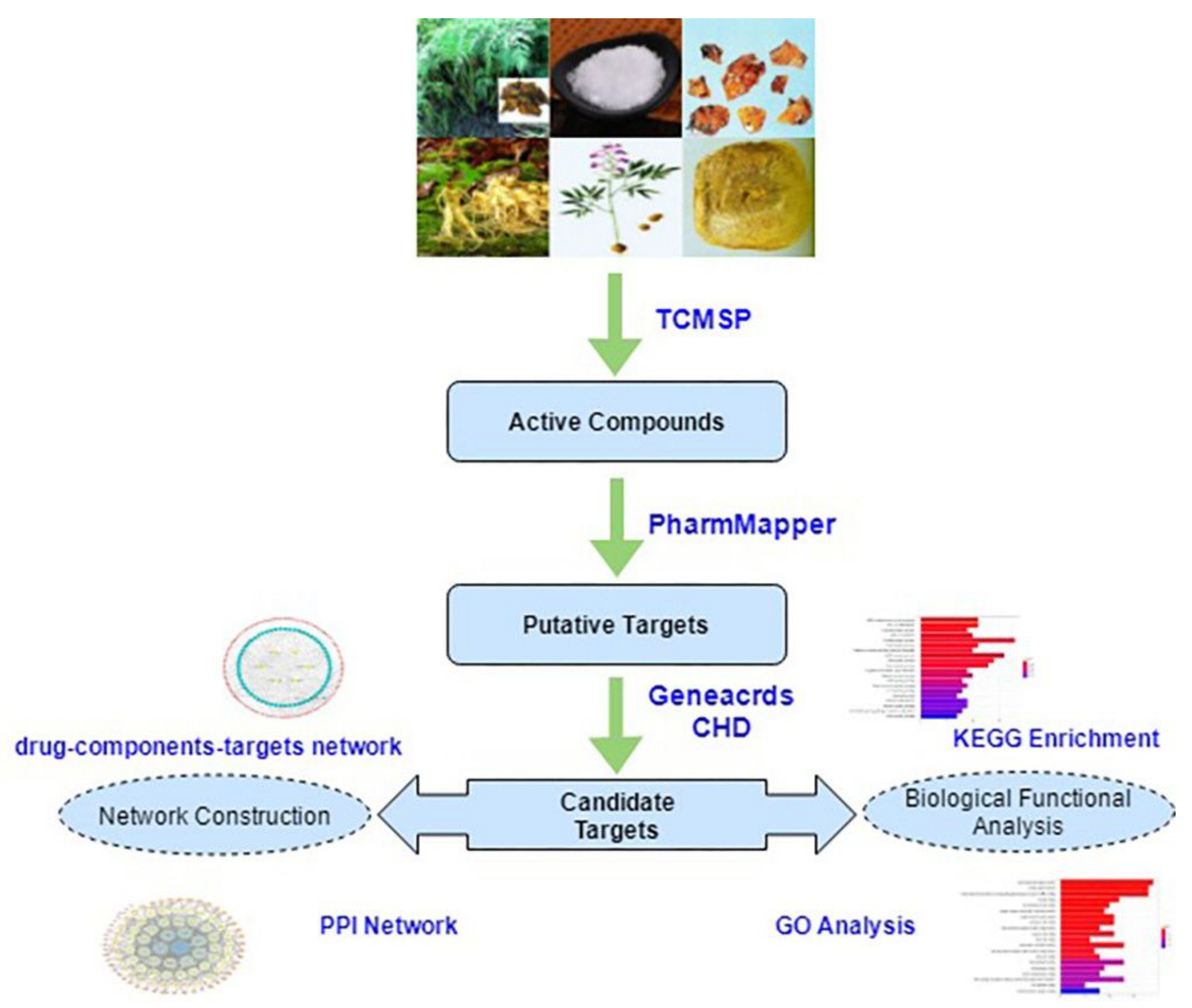

Figure 1 
The flowchart of the network pharmacology-based strategy for unraveling the mechanisms of XTN against CHD.

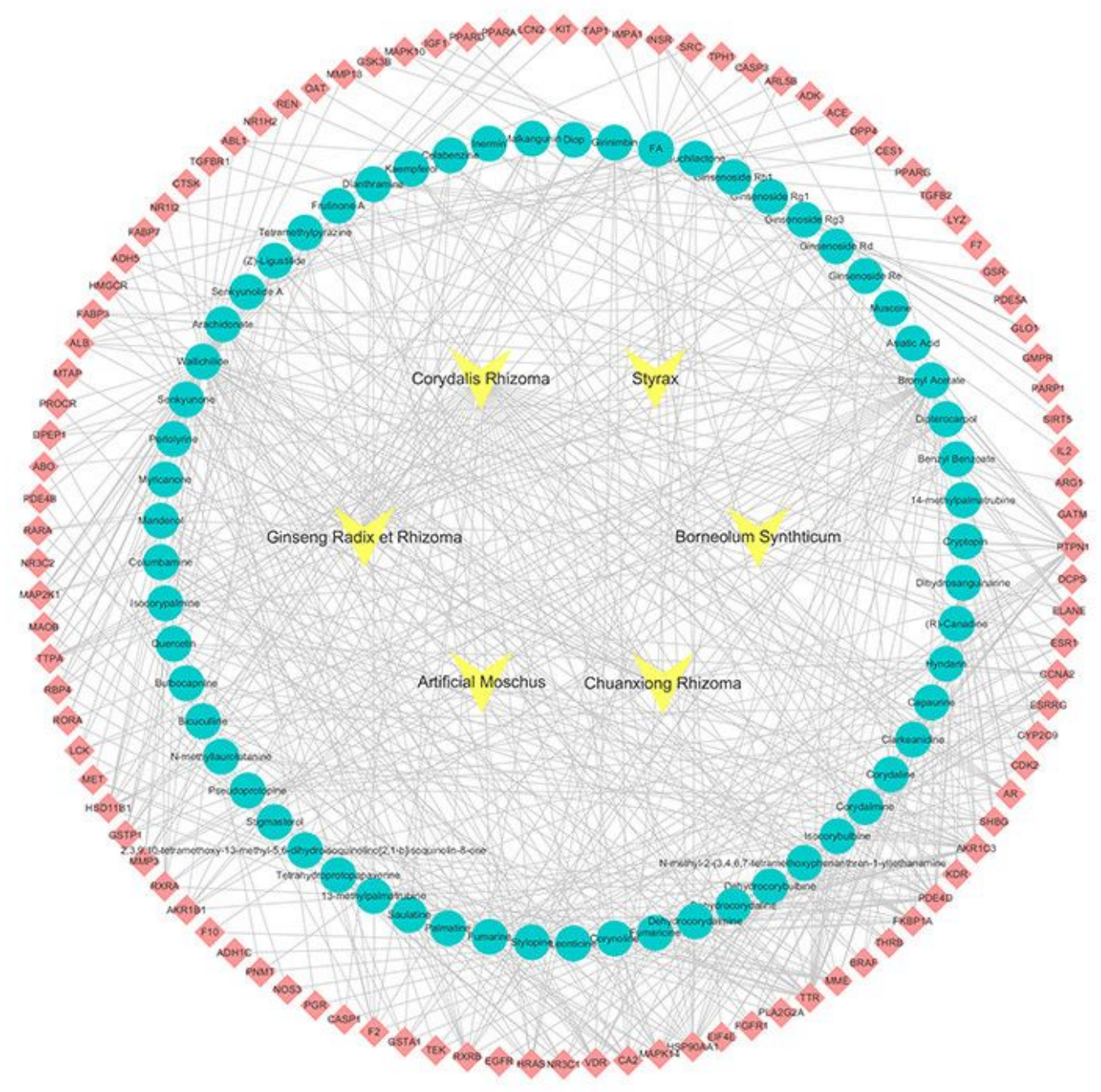

Figure 2

drug-components-targets network of 6 herbs in XTN predicted to have 104 candidate targets, the blue nodes represents chemical components and the red are candidate targets. 


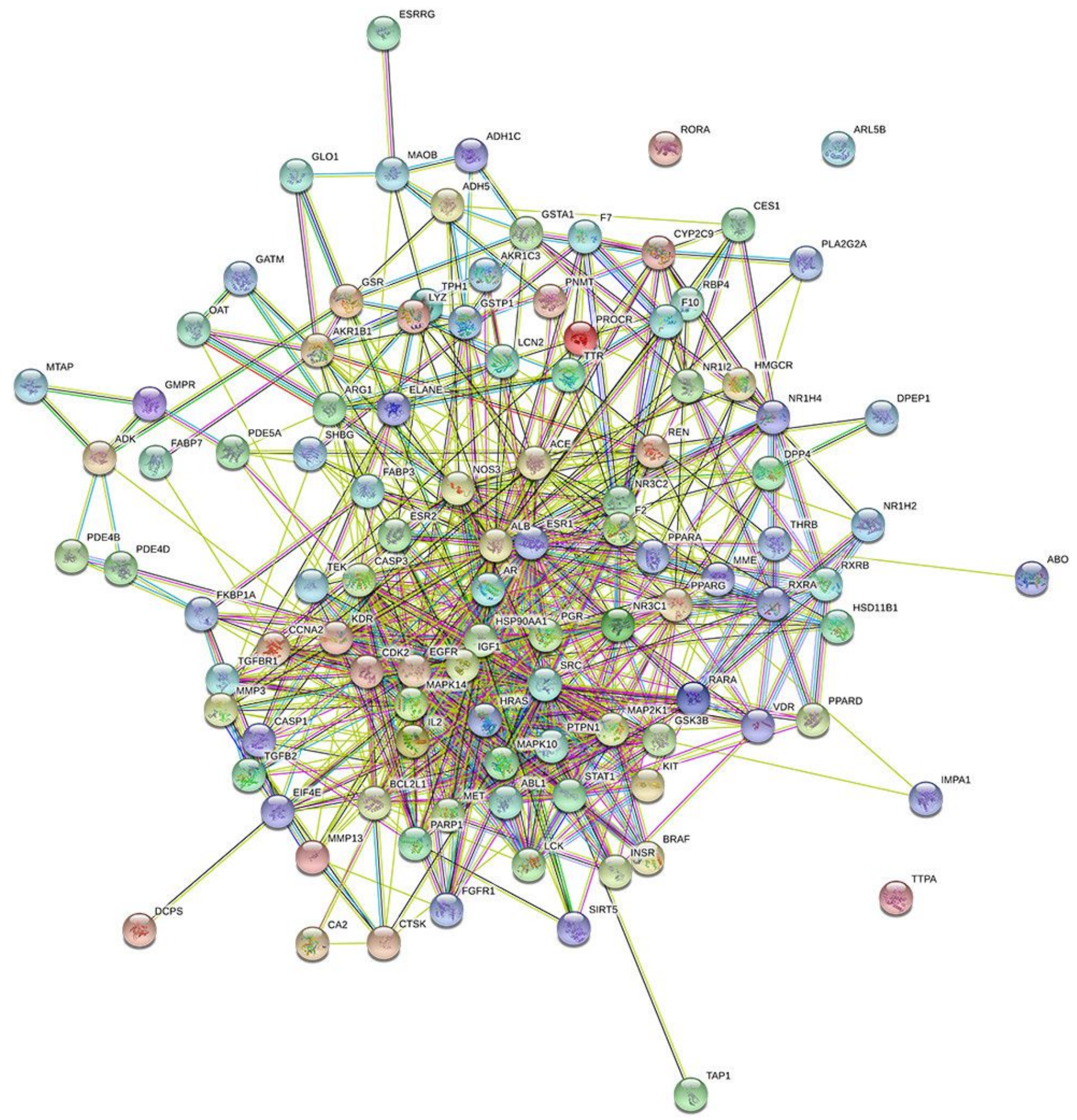

Figure 3

PPI network of XTN against CHD. 


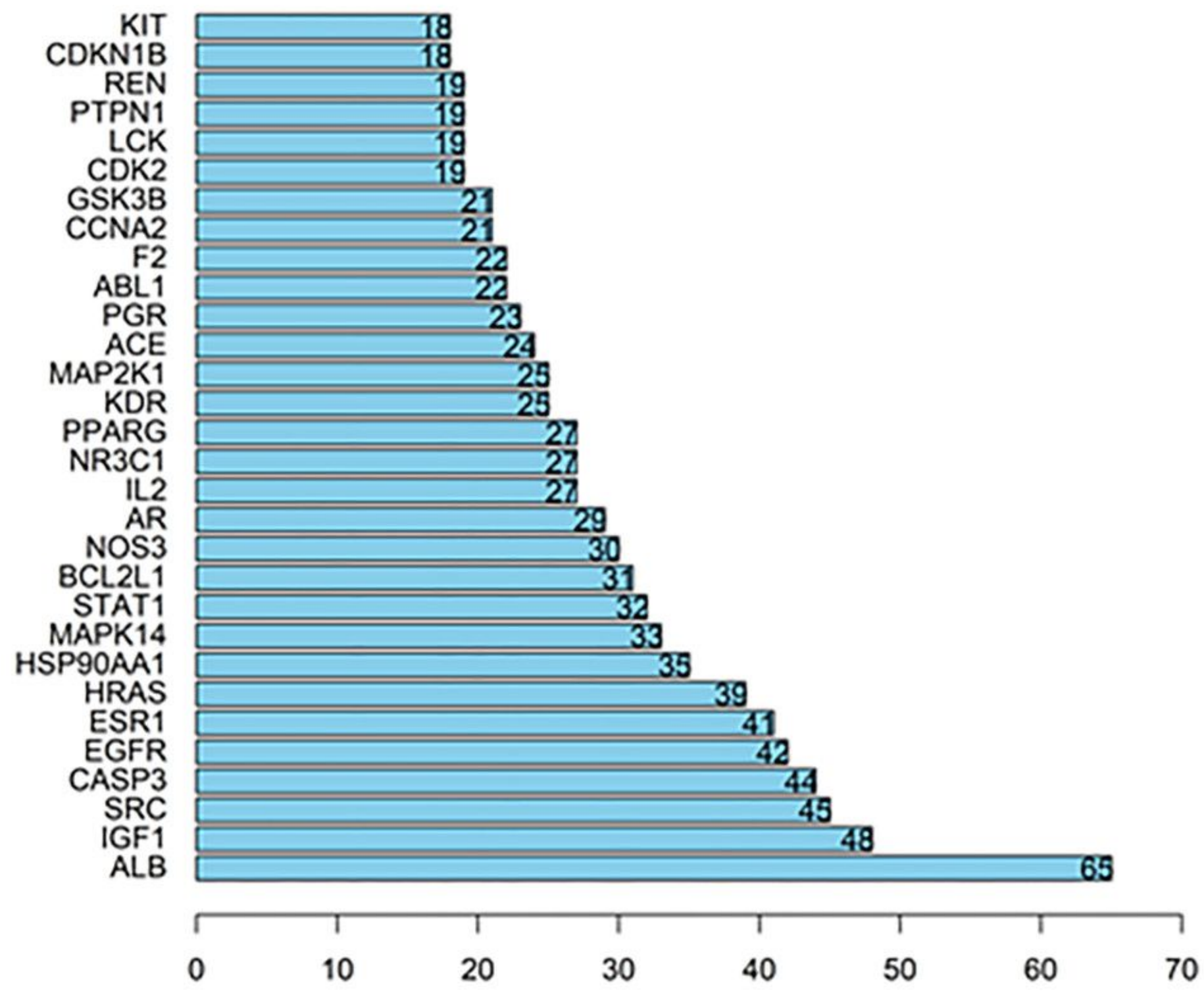

Figure 4

PPI network core proteins 


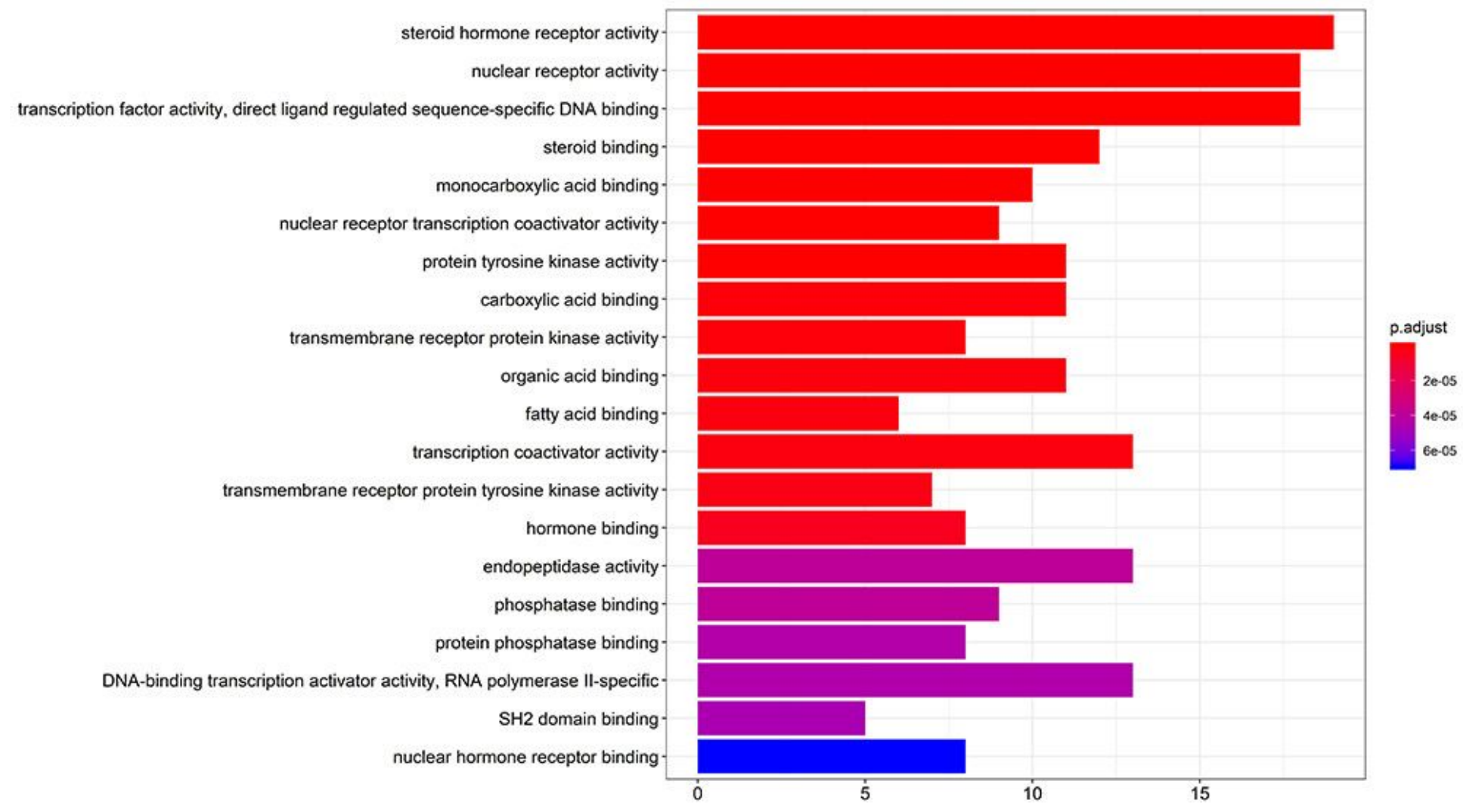

\section{Figure 5}

Top 20 of biological process enrichment. Results showed that the candidate targets involved in a variety of biological processes, such as steroid hormone receptor activity, nuclear receptor activity, transcription factor activity, director ligand regulated sequence-specific binding 


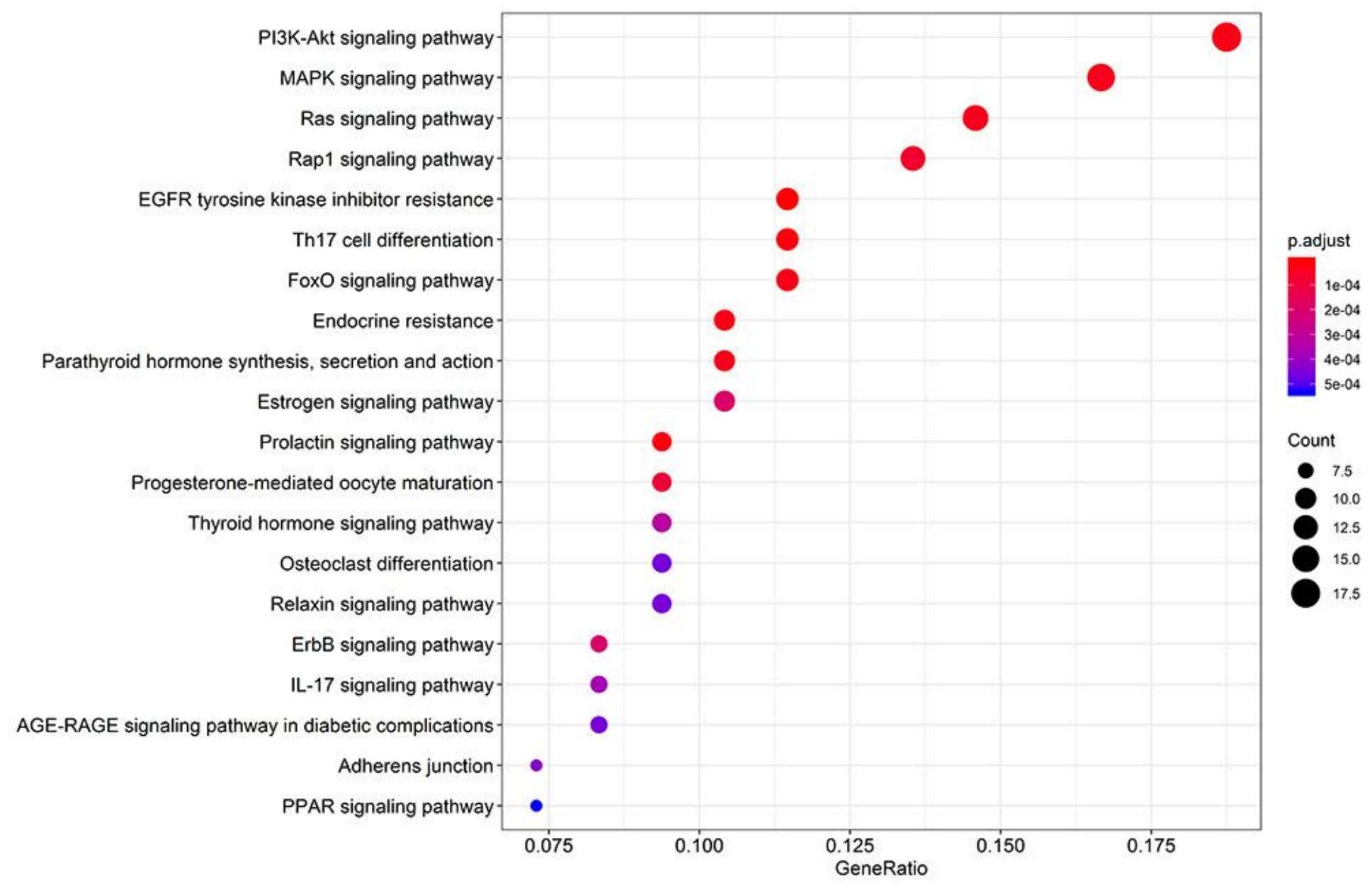

\section{Figure 6}

Top 20 of pathway enrichment. Molecular signaling pathways of XTN were highlighted, such as PI3K-AKt signaling pathway, MAPK signaling pathway, Ras signaling pathway, Rap1 signaling pathway 


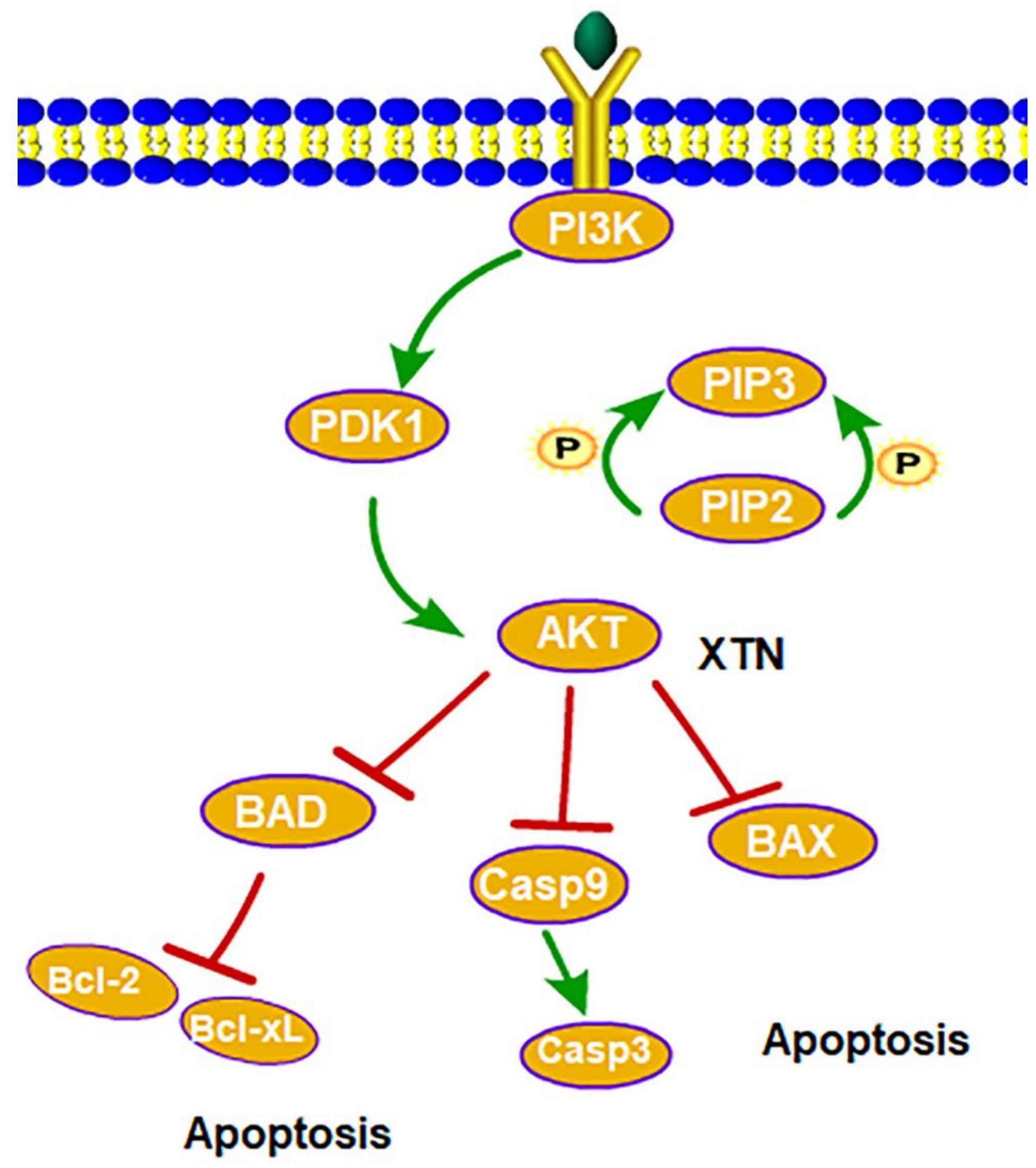

Figure 7

Illustration of the biological process PI3K-Akt pathway caused by candidate targets. 